\title{
Note on Mr. J. J. Thomson's investigation of the electromagnetic action of a moving electrified sphere
}

\section{George Francis Fitzgerald}

To cite this article: George Francis Fitzgerald (1882) Note on Mr. J. J. Thomson's investigation of the electromagnetic action of a moving electrified sphere, Philosophical Magazine Series 5 , 13:81, 302-305, DOI: $10.1080 / 14786448208627183$

To link to this article: http://dx.doi.org/10.1080/14786448208627183

冓 Published online: 28 Apr 2009.

Submit your article to this journal $[\pi$

Џll Article views: 4

Q View related articles $\llbracket$ 


\section{[ 302 ]}

\section{Intelligence and Miscellaneous Articles.}

NOTE ON MR. J. J. THOMSON'S INVESTIGATION OF THE ELECTROMAGNETIO ACTION OF A MOVING ELECTRIFIED SPHERE. BY GEORGE FRANCIS FITZGERALD, FELLOW OF TRINITY COLLEGE, DUBLIN, ETC.

TN the April number of the Philosophical Magazine for the present year Mr. J. J. Thomson has given an interesting investigation of the electromagnetic action of a moving electrified sphere.

On the fourth page (loc. cit. p. 232) of this investigation, he makes an assumption, which he does not justify, in order to make the components of the vector-potential of electromagnetic induction satisfy the condition

$$
\frac{d \mathrm{~F}}{d x}+\frac{d \mathrm{G}}{d y}+\frac{d \mathrm{H}}{d z}=0
$$

As it seemed very likely that they ought to satisfy this condition, I thought it worth while bringing before the Society a justification of his assumption, which, however, leads to slightly different equations from his, though his final result is unaffected.

Mr. Thomson has not touched the question of the discontinuity at the surface of the sphere, nor what becomes of the displacement when the sphere passes over a point. We may assume that the point remains in its displaced position (and this is practically what $\mathrm{Mr}$. Thomson assumes); but if we do, the above condition is not fulfilled. We may assume that it returns to its original position, so that no permanent displacement takes place in the track of the sphere as would occur on Mr. Thomson's assumption. This, however, does not satisfy the condition either; and I have been led to assume that the particle does return to its original position, but that in some way or other the discontinuity at the surface acts as if a moving quantity of electricity acted like an element of an electric current. This may seem like begging the question; but it is only doing explicitly what Mr. Thomson does implicitly. It is evidently impossible that the electromagnetic action of moving electricity can be due entirely to the electromagnetic action of the displacementcurrents in the dielectric; for in the case of a plane moving parallel to itself there are none of these displacement-currents, and yet that is the only case that has been experimentally verified.

To show that my assumption leads to equations satisfying the condition, and leading to practically the same results as Mr. Thomson's, does not require much work.

Consider an elementary volume $d x d s \cdot \cos \theta$, where $d s$ is an element of the surface of the sphere, and $\theta$ the angle the radius makes with $x$. The displacement in this volume is $=\delta$, the superficial density, while after the time $d t$ it is zero on my assumption.

* From the Scientific Proceedings of the Royal Dublin Society, vol. iii. part 4, having been read November 21,1881 . Communicated by the Author. 
Hence calling the displacement D, we have

Hence

$$
\frac{d \mathrm{D}}{d t} d t=-\delta \cdot d x d s \cdot \cos \theta \text {. }
$$

$$
\dot{\mathrm{D}}=-\delta \cdot \frac{d x}{d t} \cdot d s \cdot \cos \theta
$$

Now $\frac{d x}{d t}=p$, the velocity of the sphere which is supposed to be moving along $x$. Hence the components of $\dot{\mathrm{D}}$ are $\dot{f}, \dot{g}, \dot{h}$; and observing that $d s=a^{2} d \mu d \phi$, where $\cos \theta=\mu$, and $a$ is the radius of the sphere, while $4 \pi a^{2} . \delta=e$, the total quantity of electricity on the sphere,

$$
\begin{aligned}
& \dot{f} d x d y d z=-\frac{e p}{4 \pi} \mu^{2} d \mu d \phi, \\
& \dot{g} d x d y d z=-\frac{e p}{4 \pi} \mu \cos \phi d \mu d \phi, \\
& \dot{h} d x d y d z=-\frac{e p}{4 \pi} \mu \sin \phi d \mu d \phi .
\end{aligned}
$$

Hence the components of the electromagnetic potential are at a point at a distance

$$
\begin{aligned}
& \mathrm{F}_{\mathbf{s}}=-\frac{e p}{4 \pi} \iint \frac{\mu^{2}}{r} d \mu d \phi, \\
& \mathrm{G}_{\mathrm{s}}=-\frac{e p}{4 \pi} \iint \frac{\mu \cos \phi}{r} d \mu d \phi, \\
& \mathrm{H}=-\frac{e p}{4 \pi} \iint \frac{\mu \sin \phi}{r} d \mu d \phi .
\end{aligned}
$$

These are the components of the electromagnetic potential due to this superficial change of displacement that I have assumed. When integrated over the surface of the sphere, they give at a point distant $\mathrm{R}$ from its centre, and whose polar angles are $\alpha$ and $\epsilon$,

$$
\begin{aligned}
& \mathrm{F}_{\mathrm{s}}=-\frac{e p}{3 \mathrm{R}}-\frac{e p a^{2}}{5 \mathrm{R}^{3}}\left(\cos ^{2} \alpha-\frac{1}{3}\right), \\
& \mathrm{G}_{\mathrm{s}}=\quad-\frac{e p a^{2}}{5 \mathrm{R}^{3}} \cos \alpha \cdot \sin \alpha \cdot \cos e, \\
& \mathrm{H}_{\mathrm{s}}=\quad-\frac{e p a^{2}}{5 \mathrm{R}^{3}} \cos \alpha \cdot \sin \alpha \cdot \sin \epsilon .
\end{aligned}
$$

If we add to these the components calculated by Mr. Thomson as due to the external displacement-currents, and given by him (loc. cit. p. 233), namely

$$
\begin{aligned}
& \mathrm{F}_{\mathrm{e}}=\frac{e p}{10 \mathrm{R}^{3}}\left(5 \mathrm{R}^{2}-3 a^{2}\right)\left(\cos ^{2} \alpha-\frac{1}{3}\right), \\
& \mathrm{G}_{\mathrm{e}}=\frac{e p}{10 \mathrm{R}^{2}}\left(5 \mathrm{R}^{2}-3 a^{2}\right) \cos \alpha \sin \alpha \cos \epsilon, \\
& \mathrm{H}_{\mathrm{e}}=\frac{e p}{10 \mathrm{R}^{2}}\left(5 \mathrm{R}^{2}-3 a^{2}\right) \cos \alpha \sin \alpha \cos \epsilon,
\end{aligned}
$$


we get, as the resultant values of the components produced by the displacements assumed,

$$
\begin{aligned}
& \mathrm{F}_{\mathrm{d}}=-\frac{e p}{3 \mathrm{R}}+\frac{e p}{2 \mathrm{R}^{3}}\left(\mathrm{R}^{2}-a^{2}\right)\left(\cos ^{2} \alpha-3\right), \\
& \mathrm{G}_{\mathrm{d}}=\frac{e p}{2 \mathrm{R}^{3}}\left(\mathrm{R}^{2}-a^{2}\right) \cos \alpha \sin \alpha \cos \epsilon, \\
& \mathrm{H}_{\mathrm{d}}=\frac{e p}{2 \mathrm{R}^{3}}\left(\mathrm{R}^{2}-a^{2}\right) \cos \alpha \sin \alpha \sin \epsilon,
\end{aligned}
$$

which, however, do not satisfy the condition

$$
\frac{d \mathrm{~F}_{\mathrm{d}}}{d u}+\frac{d \mathrm{G}_{\mathrm{d}}}{d y}+\frac{d \mathrm{H}_{\mathrm{d}}}{d z}=0 .
$$

Now it is very easy to calculate the action of the superficial moving electricity if it be assumed to act like an electric current. Each element of the surface will act as if it had an electric $\delta . p$ on it; and the $x$ components of the electromagnetic potential will evidently be the same as the electrostatic potential, while the $y$ and $z$ components will vanish. Hence

$$
\mathrm{F}_{\mathrm{c}}=\frac{\mu e p}{\mathrm{R}}, \quad \mathrm{G}_{\mathrm{c}}=\mathrm{H}_{\mathrm{c}}=0 .
$$

$\mathrm{My}$ assumption is that the complete components are

$$
\mathrm{F}=\mathrm{F}_{\mathrm{c}}+\mathrm{F}_{\mathrm{d}}, \quad \mathrm{G}=\mathrm{G}_{\mathrm{c}}+\mathrm{G}_{\mathrm{d}}, \quad \mathrm{H}=\mathrm{H}_{\mathrm{c}}+\mathrm{H}_{\mathrm{d}} ;
$$

and it is easy to see that they can be put into the form

$$
\begin{aligned}
& \mathrm{S}=\frac{\mu e p}{\mathbf{R}}+\frac{\mu e p}{6}\left\{\left(\mathrm{R}^{2}-a^{2}\right) \frac{d^{2}}{d x^{2}} \cdot \frac{1}{\mathrm{R}}-\frac{2}{\overline{\mathrm{R}}}\right\}, \\
& \mathrm{G}=\quad \frac{\mu e p}{6}\left(\mathrm{R}^{2}-a^{2}\right) \frac{d^{2}}{d x d y} \cdot \frac{1}{\mathbf{R}}, \\
& \mathrm{H}=\quad \frac{\mu e p}{6}\left(\mathrm{R}^{2}-a^{2}\right) \frac{d^{2}}{d x d z} \cdot \frac{1}{\mathbf{R}} ;
\end{aligned}
$$

and these satisfy the condition

$$
\frac{d \mathrm{~F}}{d x}+\frac{d \mathrm{G}}{d y}+\frac{d \mathrm{H}}{d z}=0 .
$$

These may be further simplified by assuming a function

$$
\begin{aligned}
& \mathrm{X}=-\frac{\mu e \underline{p}}{2}\left(x+a^{\varepsilon} \frac{d}{d x}\right) \frac{1}{\mathrm{R}}, \\
& \mathrm{F}=-\frac{\mu e \underline{p}}{\mathrm{R}}+\frac{d \chi}{d x}, \\
& \mathrm{G}=\frac{d \chi}{d y}, \\
& \mathrm{H}=\frac{d \chi}{d z} .
\end{aligned}
$$


We hence easily see that, in the general case where the sphere is moving with component velocities $p, q, r$, we must take

or, calling

$$
\chi=-\frac{\mu e}{z}\left\{p x+q y+r z+a^{2}\left(p \frac{d}{d x}+q \frac{d}{d y}+r \frac{d}{d z}\right)\right\} \frac{1}{\mathbf{R}} ;
$$

and observing that

$$
p \frac{d}{d x}+q \frac{d}{d y}+r \frac{d}{d z}=\frac{d}{d \theta}
$$

$$
p \frac{x}{\mathbf{R}}+q \frac{y}{\mathrm{R}}+r \frac{z}{\overline{\mathrm{R}}}=\dot{\mathrm{R}},
$$

we have

$$
\chi=-\frac{\mu e}{2}\left(\dot{\mathrm{R}}+a^{2} \frac{d}{d \theta} \cdot \frac{1}{\mathrm{R}}\right)
$$

and then we have

$$
\begin{aligned}
& \mathrm{F}=\frac{\mu e p}{\mathbf{R}}+\frac{d \chi}{d x}, \\
& \mathrm{G}=\frac{\mu e q}{\overline{\mathrm{R}}}+\frac{d \chi}{d y}, \\
& \mathrm{H}=\frac{\mu e r}{\mathbf{R}}+\frac{d \chi}{d z} .
\end{aligned}
$$

From these it appears at once that the magnetic effect of the displacement-currents is nit. For the components of the magnetic forces $\alpha, \beta, \gamma$ are

$$
\begin{aligned}
& \alpha=\frac{d \mathrm{G}}{d z}-\frac{d \mathrm{H}}{d y}, \\
& \beta=\frac{d \mathrm{H}}{d x}-\frac{d \mathrm{~F}}{d z}, \\
& \gamma=\frac{d \mathrm{~F}}{d y}-\frac{d \mathrm{G}}{d x}
\end{aligned}
$$

and $\chi$ disappears from this, and there only remains the magnetic action due to the current that I have assumed to represent the moving superficial electricity. The effect of this is obviously the same as if the whole quantity of electricity were moving at its centre; and this is the same as Mr. Thomson's result (l. c. p. 236).

It is to be observed that Mr. Thomson has intentionally omitted the self-induction of these displacement-currents on one another; and it may legitimately be omitted when the motion is comparatively slow; but a complete solution of the question would be most interesting.

It may be worth while remarking, that no effect except light has ever yet been traced to the displacement-currents assumed by Maxwell in order to be able to assume all currents to flow in closed circuits. It has not, as far as I am aware, been ever actually demonstrated that open circuits, such as Leyden-jar discharges, produce exactly the same effects as closed circuits; and until some such effect of displacement-currents is observed. the whole theory of them will be open to question. 